\title{
DNA copy number concentration measured by digital and droplet digital quantitative PCR using certified reference materials
}

\author{
Philippe Corbisier • Leonardo Pinheiro • Stéphane Mazoua • \\ Anne-Marie Kortekaas • Pui Yan Jenny Chung • Tsvetelina Gerganova • \\ Gert Roebben • Hendrik Emons • Kerry Emslie
}

Received: 21 November 2014 /Revised: 19 December 2014 / Accepted: 5 January 2015 /Published online: 20 January 2015

(C) The Author(s) 2015. This article is published with open access at Springerlink.com

\begin{abstract}
The value assignment for properties of six certified reference materials (ERM-AD623a-f), each containing a plasmid DNA solution ranging from 1 million to 10 copies per $\mu \mathrm{L}$, by using digital PCR (dPCR) with the BioMark ${ }^{\mathrm{TM}}$ HD System (Fluidigm) has been verified by applying droplet digital PCR (ddPCR) using the QX100 system (Bio-Rad). One of the critical factors in the measurement of copy number concentrations by digital PCR is the partition volume. Therefore, we determined the average droplet volume by optical microscopy, revealing an average droplet volume that is $8 \%$ smaller than the droplet volume used as the defined parameter in the QuantaSoft software version 1.3.2.0 (Bio-Rad) to calculate the copy number concentration. This observation explains why copy number concentrations estimated with ddPCR and using an average droplet volume predefined in the QuantaSoft software were systematically lower than those measured by dPCR, creating a significant bias between the values obtained by these two techniques. The difference was not significant anymore when the measured droplet volume of $0.834 \mathrm{~nL}$ was used to estimate copy number concentrations. A new version of QuantaSoft software (version 1.6.6.0320), which has since been released with Bio-Rad's new QX200 systems and QX100 upgrades, uses a droplet volume of $0.85 \mathrm{~nL}$ as a defined parameter to calculate copy number concentration.
\end{abstract}

Published in the topical collection Nucleic Acid Quantification with guest editors Hendrik Emons and Philippe Corbisier.

P. Corbisier $(\bowtie) \cdot$ S. Mazoua $\cdot$ A.-M. Kortekaas $\cdot$ P. Y. J. Chung $\cdot$

T. Gerganova $\cdot$ G. Roebben $\cdot$ H. Emons

Institute for Reference Materials and Measurements, Joint Research

Centre, European Commission, Retieseweg 111, 2440 Geel, Belgium

e-mail: philippe.corbisier@ec.europa.eu

L. Pinheiro $\cdot$ K. Emslie

Bioanalysis Group, National Measurement Institute, 1 Suakin Street,

Pymble, New South Wales 2066, Australia
Keywords Digital PCR · Droplet digital PCR · Reference materials · Optical microscopy $\cdot$ Areal equivalent diameter

\section{Introduction}

Quantification of nucleic acid molecules by quantitative PCR (qPCR) has been developed during the last 25 years in many applications of green and red biotechnologies and has progressively reached a level of maturity [1]. qPCR has been applied to gene expression quantification $[2,3]$, forensic DNA quantification $[4,5]$ and clinical $[6,7]$ and veterinary [8] virology diagnostics, all reviewed recently.

Quantification of nucleic acids by qPCR is commonly achieved by using a calibration curve, constructed by measuring the number of cycles required for the fluorescent signal to reach a threshold level for known amounts of target DNA in a parallel set of reactions. The threshold fluorescent level is calculated from the initial cycles, and this cycle number $(\mathrm{Cq}$ value) is proportional to the number of copies of template in the sample. The use of calibration curves has basically two consequences.

First, it requires that the exact quantity value carried by a calibration material (measurement standard) is determined by independent means using, e.g. spectrophotometry or an intercalating dye. Spectrophotometric methods rely on the molar absorptivity (or the molar absorption coefficient) of pure nucleic acid solutions used in the equation based on the Lambert-Beer law [9]. The molar absorptivity depends upon the chemical nature (deoxyribonucleic or ribonucleic) and conformation (single or double stranded) of the nucleic acid molecule as well as its degree of purity. The fraction of the absorbed light will depend on how many molecules the light interacts with, being the molecule of interest but also 
contaminants that have been co-extracted. Despite those limitations, spectrophotometric methods are widely used to quantify nucleic acids in solutions. Fluorometric methods using intercalating dyes have as a major advantage their higher sensitivity compared to spectrophotometric methods, allowing a detection down to $25 \mathrm{pg} / \mathrm{mL}$ dsDNA [10]. However, these measurements need to be calibrated using the quantity value of a material having ideally the same chemical nature and molecular weight as the analyte of interest. This circular problem cannot be solved without the use of a reference material that is certified for its nucleic acid concentration, a reason why several metrology institutes are developing such materials.

The use of an external measurement standard being an amplicon [11], cut or uncut plasmid DNA [12, 13], cosmid [14] or complementary DNA [15] to calibrate qPCR has a second drawback. A number of strategies have been used to prepare and apply a standard with similar properties to the target DNA. These include treating genomic DNA with a cocktail of restriction enzymes, DNA ultrasonication [16], shredding methods [17] or using a Bayesian approach that takes into account several sources of uncertainty [18]. A few studies have shown that those standards could behave in a similar way as the nucleic acid molecules that were targeted $[19,20]$. However, factors such as DNA stability, base composition, secondary structure and presence of complex mixtures of non-target DNA can significantly alter the PCR amplification performance, making the DNA quantification dependent and only traceable to the calibrants that have been used [21].

Digital PCR (dPCR) technology [22] is an end-point measurement that overcomes the dependency on a DNA calibrant, and it has recently been used as an alternative method to assign copy number concentrations of defined nucleic acids in solution [23-26]. Some prerequisites of the technology have already been discussed [27], and recommendations were published on how to properly report digital PCR results [28]. The nucleic acid targets must be randomly distributed among the partitions, and the conditions of the PCR assays must be optimised to ensure that a single copy of the target is indeed amplified during the PCR. Since the calculation of the copy number concentration includes dividing the copy number estimate by the assay volume, knowing the correct partition or droplet volume is a key factor when measuring DNA concentrations using digital PCR [29]. The partition or droplet volumes as provided by the manufacturer are utilised by the majority of laboratories without further independent verification. The potential error on that volume is not important if a ratio between two concentrations of DNA targets is reported, but it can generate a significant bias when an absolute copy number concentration is measured.

In this study, we used a set of reference materials that has been certified by using dPCR for the absolute copy number concentration of a $B C R-A B L$ target $[24,30]$ applying the BioMark $^{\mathrm{TM}}$ HD System (Fluidigm). The certified concentrations were verified using results from the QX100 droplet digital PCR (ddPCR) system (Bio-Rad). In parallel, the average droplet volume was measured and the copy number concentration reported by the ddPCR QuantaSoft software (Bio-Rad) was corrected by taking into account the real average droplet volume.

\section{Materials and methods}

Test material

ERM-AD623a, ERM-AD623b, ERM-AD623c, ERMAD623d, ERM-AD623e and ERM-AD623f (Joint Research Centre-Institute for Reference Materials and Measurements (JRC-IRMM)) were used as certified reference materials containing $\left(1.08 \times 10^{6} \pm 0.13 \times 10^{6}\right),\left(1.08 \times 10^{5} \pm 0.11 \times 10^{5}\right),(1.03 \times$ $\left.10^{4} \pm 0.10 \times 10^{4}\right),\left(1.02 \times 10^{3} \pm 0.09 \times 10^{3}\right),\left(1.04 \times 10^{2} \pm 0.10 \times\right.$ $\left.10^{2}\right)$ and $(10.0 \pm 1.5)$ copies $(\mathrm{cp}) / \mu \mathrm{L}$, respectively, of a doublestranded linearised plasmid [24]. The plasmid is in a 1-mmol/L Tris, 0.01-mmol/L EDTA pH 8.0 buffer $\left(\mathrm{T}_{1} \mathrm{E}_{0.01}\right)$ supplemented with $50 \mathrm{mg} / \mathrm{L}$ of transfer RNA from Escherichia coli and used undiluted in the ddPCR experiment unless otherwise mentioned. The copy number concentrations and related uncertainties were established using the measurement data of three (National) Metrology Institutes each using their own dPCR apparatus from Fluidigm (i.e. the BioMark ${ }^{\mathrm{TM}}$ HD System) and two validated assays as described earlier [24].

\section{Digital PCR}

Eppendorf DNA LoBind tubes (VWR International, cat no. 0030108051, Leuven, Belgium) as well as calibrated Rainin Pipet-Lite XLS+ single channel micropipettes (Mettler-Toledo S.A., Zaventem, Belgium) were used throughout this study. For the measurement of copy number concentrations of the six plasmid solutions by dPCR, $10 \mu \mathrm{L}$ of ERM-AD623a, ERMAD623b, ERM-AD623c and ERM-AD623d was gravimetrically diluted in $\mathrm{T}_{1} \mathrm{E}_{0.01}$ buffer to obtain DNA samples at a nominal concentration of $500 \mathrm{cp} / \mu \mathrm{L}$. ERM-AD623e and ERM-AD623f were used undiluted in the dPCR assay. A volume of $19.7 \mu \mathrm{L}$ of the DNA sample was further mixed with $30.3 \mu \mathrm{L}$ of pre-sample mix solution, and $9 \mu \mathrm{L}$ of this mixture was loaded on five panels of the 12.765 digital Array ${ }^{\mathrm{TM}}$ IFCs from Fluidigm (BIOKE, Leiden, Netherlands). The presample mix solution contained the primers and probes for the $B C R-A B L$ b3a2 transcript and for the $A B L$ transcript (duplex PCR conditions) at final concentrations mentioned in Table 1 together with $20 \times$ GE sample loading reagent (Fluidigm) and TaqMan ${ }^{\circledR}$ Universal PCR MasterMix (Applied Biosystems, Ghent, Belgium) as recommended by the 
manufacturer. The PCR was performed according to the specifications listed in Table 2. The PCR runs were then analysed with the Fluidigm Digital PCR software version 3.0.2 using the following settings: quality threshold 0.4 ; linear baseline correction; automatic $\mathrm{Ct}$ threshold method; and target $\mathrm{Ct}$ range between 20 and 49. In case of low background noise, the results were analysed using a linear derivative baseline correction and a manual $\mathrm{Ct}$ threshold setting.

\section{Droplet digital PCR}

The copy number concentrations of the six plasmid solutions were measured by ddPCR using the QX100 ${ }^{\mathrm{TM}}$ Droplet Digital $^{\text {TM }}$ PCR system (Bio-Rad, Temse, Belgium). A volume of $33.6 \mu \mathrm{L}$ was pipetted from each CRM vial and mixed with $62.4 \mu \mathrm{L}$ pre-sample solution. The pre-sample solution contained $48 \mu \mathrm{L}$ of ddPCR Supermix (Bio-Rad, cat no. 1863010), $4.8 \mu \mathrm{L}$ forward and reverse primers and the probe for the $B C R-A B L$ b3a2 transcript at the same final concentrations as those used for the dPCR experiments. ERM-AD623f, e and $\mathrm{d}$ were used undiluted and ERM-AD623c, b and a were gravimetrically diluted with $\mathrm{T}_{1} \mathrm{E}_{0.01}$ to a nominal concentration of $1000 \mathrm{cp} / \mu \mathrm{L}$. Twenty microliters of this solution was pipetted in eight compartments of the Droplet Generator DG8 Cartridge (Bio-Rad, cat no. 186-3008) and droplets were generated. The entire droplet emulsion volume was further loaded in a semi-skirted and PCR-clean 96-well PCR plate (Eppendorf, Leuven, Belgium). The loaded 96-well PCR plate was then heat sealed with pierceable foil in the PX1TM PCR Plate Sealer and placed in a C1000 Touch ${ }^{\mathrm{TM}}$ Thermo Cycler (both from Bio-Rad). The same thermal cycling conditions were applied as those used for dPCR (described in Table 2) except that the UNG step was omitted and only 40 PCR cycles were run. After PCR amplification, the droplets were analysed in a QX100" droplet reader (Bio-Rad), and the absolute quantification of PCR targets was analysed using QuantaSoft ${ }^{\mathrm{TM}}$ software version 1.3.2.0 with a threshold placed at an amplitude between 3000 and 4002 .

The copy number concentration $\left(T_{\mathrm{c}}\right)($ in $\mathrm{cp} / \mu \mathrm{L})$ was also calculated using Eq. (1) taking into account the number of
Table 2 Thermal cycle protocol used for both $\mathrm{dPCR}$ and ddPCR protocols

\begin{tabular}{llrlc}
\hline Name & Phase & Time $[\mathrm{s}]$ & Temperature $\left[{ }^{\circ} \mathrm{C}\right]$ & Repeats \\
\hline \multirow{2}{*}{ UNG and hot start } & UNG & 120 & 50 & 1 \\
\multirow{4}{*}{ PCR cycles } & Hot start & 600 & 95 & \\
& Denaturation & 15 & 95 & 50 \\
& Annealing & 60 & 60 &
\end{tabular}

droplets analysed $(C)$, the number of positive droplets $(H)$, the average volume of a droplet determined experimentally $\left(V_{\mathrm{d}}\right.$ in $\left.\mu \mathrm{L}\right)$ and the final dilution factor of the sample $\left(D_{\mathrm{f}}\right)$.

$T_{\mathrm{c}}=D_{\mathrm{f}} \times\left(\frac{1}{C \times V \mathrm{~d}}\right) \times \frac{\left(\log \left(1-\frac{H}{C}\right)\right)}{\left(\log \left(1-\frac{1}{C}\right)\right)}$

The final dilution $\left(D_{\mathrm{f}}\right)$ was calculated using Eq. (2) as the product of the dilution factor of the sample $\left(D_{\mathrm{s}}\right)$ (if any) and the dilution factor of the DNA solution in the assay $\left(D_{\mathrm{a}}\right)$.

$D_{\mathrm{f}}=D_{\mathrm{s}} \times D_{\mathrm{a}}$

The volume of each sample in the assay was determined by dividing the sample mass $\left(m_{\mathrm{DNA} \text { sol }}\right)$ by the density of the solution $\left(\delta_{\mathrm{DNA} \text { sol }}\right)$. The density of the pre-sample mix $\left(\delta_{\mathrm{p} \mathrm{sol}}\right)$ was determined by weighing 10 times $100 \mu \mathrm{L}$ of the presample mix $\left(m_{\mathrm{p} \text { sol }}\right)$ on a ME235P analytical microbalance (Sartorius, Vilvoorde, Belgium). Each mass was divided by the pipetted volume to calculate the density. A density of $1.03532 \mathrm{~g} / \mathrm{mL}$ for the pre-sample mix $\left(\delta_{\mathrm{p} \text { sol }}\right)$ and a density of $1.0000 \mathrm{~g} / \mathrm{mL}$ for the sample $\left(\delta_{\text {DNA sol }}\right)$ were taken into account in the calculation of the dilution factor in the assay $\left(D_{\mathrm{a}}\right)(\mathrm{Eq} .3)$.

$D_{\mathrm{a}}=\frac{\frac{m_{\text {DNA sol }}}{\delta_{\text {DNA sol }}}+\frac{m_{\mathrm{p} \mathrm{sol}}}{\delta_{\mathrm{p} \mathrm{sol}}}}{\frac{m_{\text {DNA sol }}}{\delta_{\text {DNA sol }}}}$

Table 1 Primers and probes used to amplify the $B C R-A B L$ b3a2 transcript by dPCR and ddPCR

\begin{tabular}{lllll}
\hline PCR assay & Primers and probe & Sequence & $\begin{array}{l}\text { Final concentration } \\
{[\mu \mathrm{M}]}\end{array}$ & $\begin{array}{l}\text { Amplicon size } \\
{[\mathrm{bp}]}\end{array}$ \\
\hline$B C R-A B L$ b3a2 & F-primer & 5'-TCCGCTGACCATCAAYAAGGA-3' & 0.3 & 149 \\
& R-primer & 5'-CACTCAGACCCTGAGGCTCAA-3' & 0.3 & 0.2 \\
\multirow{2}{*}{$A B L$} & Probe & 5'-(6-VIC)CCCTTCAGCGGCCAGTAGCATCTGA-(MGB)-3' & 0.3 & 122 \\
& F-primer & 5'-TGGAGATAACACTCTAAGCATAACTAAAGGT-3' & 0.3 & \\
& R-primer & 5'-GATGTAGTTGCTTGGGACCCA-3' & \\
\hline
\end{tabular}


Droplet volume sizing by optical microscopy

Average droplet volumes were determined for three DG8-1863008 cartridges, each prepared on a different day. Droplet generation and acquisition of the optical microscopy images was performed on the same day. Image analysis was performed afterwards, off-line. For each of the three cartridges, a volume of $10 \mu \mathrm{L}$ containing the droplets generated in the DG8-186-3008 cartridges was carefully pipetted into the chamber of an Ibidi plate (Ibidi $\mu$-slide VI-flat non-coated, Proxylab sprl, Beloeil, Belgium). The Ibidi plates were held at an angle to allow the formation of a uniform monolayer of droplets for better imaging. Four wells were randomly selected from each of the three different droplet generator cartridges for this analysis. Between 140 and 160 droplets were measured in each selected well. Each cartridge was analysed on a different day providing measurement of 1794 droplets in total.

An optical microscope (Leica DM 4000M, Diegem, Belgium) with a digital CCD camera (Leica DFC 290 HD) was used to image droplets in four wells, randomly selected from each of the three different droplet generator cartridges. All images were recorded under uniform illumination in a bright field imaging mode, using sufficient magnification $(\times 200)$. The accuracy of the scale of the images was verified with a calibration grid (Pyser-SGI Ltd, Edenbridge, UK).

The images were analysed by using ImageJ automated image analysis software (v1.47q) following an existing procedure described by Pinheiro et al. [29]. The images were first converted to a bit depth of 8 bits for image processing. The edges of the droplets were identified using the 'find edges' algorithm followed by thresholding. To enable detection of the full droplet, a 'fill holes' algorithm was run after noise reduction based on a despeckling function. The watershed algorithm implemented in the ImageJ software was used to separate touching droplets. Droplets on the edge of the images were excluded from the analysis. Between 140 and 160 droplets were measured in each of the 12 selected wells providing measurement of 1794 droplets in total.

Several approaches can be followed to measure the 3D droplet volume from the 2D images. One approach is described in detail in reference [29]: the major and minor axes of an elliptical fit to the droplet outline are determined and used to calculate the area (in pixels) of an ellipse of equivalent dimensions. This value was then used to determine the diameter of a circle of an equivalent area, and finally, the volume of a droplet was calculated assuming it has the shape of a perfect sphere. The second approach consists of measuring the average Feret diameter of the droplets as the average of their maximum and minimum Feret diameter. This value can be used as a direct estimate of the diameter of the individual droplets [31].

The length scale of the microscope used at IRMM was calibrated with a calibration grid, leading to an estimated calibration uncertainty of $0.15 \%$. The National Measurement Institute of Australia (NMIA) produced a more detailed type B uncertainty evaluation of the individual droplet volume measurement via the area equivalent diameter and estimated the relative expanded uncertainty of this measurement as $2.0 \%$. This uncertainty includes factors such as operator bias in the imaging process, calibration, off-focus image and a component for the (minor) non-sphericity of the droplets.

\section{Results}

Concentration measurements using $\mathrm{dPCR}$

In the first experiment, the comparability of results obtained by two different dPCR platforms (chip vs. droplet based) was investigated by measuring the six plasmid solutions present in the ERM-AD623 set of certified reference materials. A volume of $8.5 \mu \mathrm{L}$ of each of the six concentrations ranging from $1.08 \times 10^{6}$ to $10 \mathrm{cp} / \mu \mathrm{L}$ was tested in eight replicates in ddPCR. In Fig. 1, the highest concentration was diluted $10 \times$ in a 1$\mathrm{mmol} / \mathrm{L}$ Tris, $0.01-\mathrm{mmol} / \mathrm{L}$ EDTA $\mathrm{pH} 8.0$ buffer to avoid an overloading of positive droplets. The conditions of the PCR assay were optimal as the population of positive droplets could clearly be discriminated from the negative droplets using a threshold value of 4002 .

The certified values as determined by $\mathrm{APCR}$ were plotted against the average copy number concentration determined by ddPCR using the QuantaSoft software version 1.3.2.0 (Fig. 2). A linear regression fitted the data with a coefficient of determination of 0.9994 in a log-log plot suggesting good agreement between the two digital PCR methodologies based on chip partitions and droplets.

The apparently good agreement between the certified values and the measured values was verified by a structured and quantitative approach that allows making a statement on the evidence of any bias. The approach takes into account the certified value and its uncertainty, the measurement result and their respective uncertainties. These uncertainties were subsequently combined, and the expanded uncertainty was compared to the difference between the measured values and the certified values as explained in [32] and detailed in Table 3.

The copy number concentration of the plasmid measured by ddPCR appeared to be slightly underestimated when compared to the certified values. A statistically significant bias (at $95 \%$ confidence level) varying between 14 and $17 \%$ was observed for ERM-AD623a, ERM-AD623b, ERM-AD623c, ERM-AD623d and ERM-AD623e corresponding to the copy number concentration level ranging from 1,080,000 to $104 \mathrm{cp} /$ $\mu \mathrm{L}$. No significant bias was observed for the lowest concentration certified at $10 \mathrm{cp} / \mu \mathrm{L}$. 
Fig. 1 Discrimination of the positive and negative droplets using the $B C R-A B L$ ddPCR assay on the ERM-AD623 reference material series. The positive droplets are represented in green, whereas the negative droplets are coloured in grey

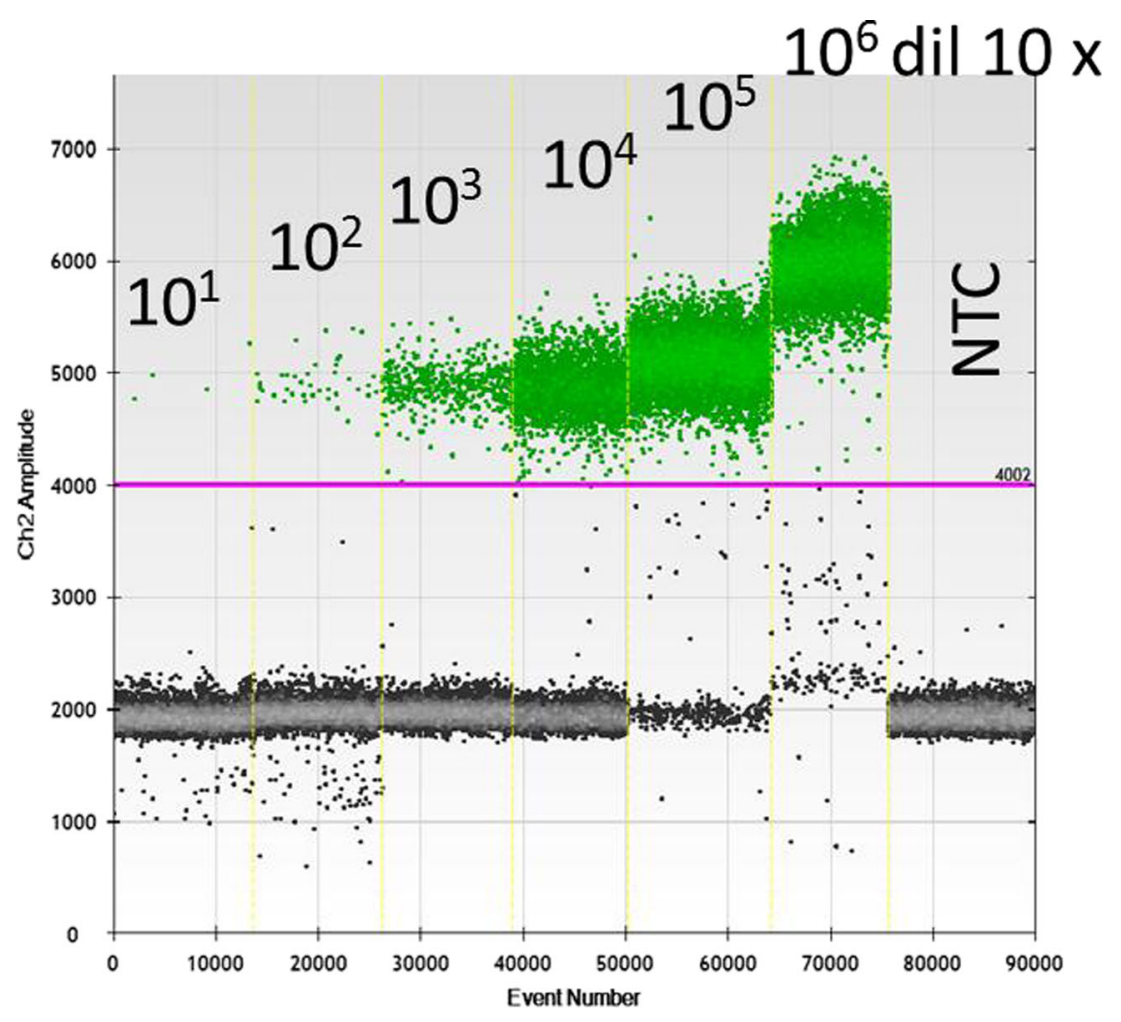

Underestimation of copy number concentration can have several origins, related either to the PCR assay itself, to the density value used for the gravimetric dilution, to the volume of the assay or to a combination of those three factors. The PCR assays used in this study have been optimised to reduce to a minimum the rain effect, so that the setting of a threshold value to segregate positive droplets from negative droplets was not an issue. Indeed both populations of droplets were clearly segregated (Fig. 1). The plasmid solutions used as test materials were small linearized plasmid to reduce the false negative droplets that may occur with larger unrestricted DNA molecules. Special care was also taken to always dilute the DNA in buffered solutions to reduce potential degradation of the targets prior amplification, reducing as such the probability to miss some droplets containing the target of interest. The PCR platform used was a C1000 Touch Thermo Cycler recommended by Bio-Rad for having uniform, slow ramping temperatures through the plate reducing border plate effects and particularly adapted for this digital PCR application. Special care was also taken to carefully correct the gravimetric dilutions using the density of the pre-mix. Having taken all those precautions, one obvious parameter which is the final volume of the assay had to be investigated.

Therefore, the hypothesis that the observed bias could be the result of an incorrect average droplet volume used in the QuantaSoft software to convert positive droplets into a copy number concentration was tested in subsequent experiments.
Droplet volume measurements using optical microscopy

To test the above hypothesis, ERM-AD623c, certified to contain $10,300 \pm 1000 \mathrm{cp} / \mu \mathrm{L}$ was measured by ddPCR under repeatability and intermediate repeatability conditions by analysing samples from three vials in three independent experiments $(N=3)$ in eight replicates $(n=8)$. The droplets generated in the DG8-138-3008 cartridges were examined under an optical microscope and simultaneously analysed in the droplet reader.

In order to avoid that the examination under the microscope would alter the size of the droplets, the photography of the generated droplets was done in less than $30 \mathrm{~min}$. (The manufacturer recommends starting thermal cycling within $30 \mathrm{~min}$ of sealing the PCR plate.)

Over the 3 days, a total of 1794 droplets were analysed and imaged by optical microscopy to calculate an average droplet volume. A typical image of a droplet layer is provided in Fig. $3 \mathrm{~A}$ together with the image obtained after the image analysis in Fig. 3B. For reasons of surface energy minimisation, the liquid droplets in the liquid (oil) matrix have a strong tendency to take a perfectly spherical shape. This shape could be distorted under the action of mechanical stress, for example due to gravity, but during the microscopy investigation, the droplets were submersed in oil, and therefore, the spherical shape is not strongly challenged in the vertical direction. Also, as shown in Fig. 3, there is no indication that the interaction between neighbouring particles results in lateral deformation. 
Fig. 2 Comparison of both digital PCR platforms using the certified reference material ERMAD623 as test material.

Horizontal error bars represent the expanded uncertainty associated to the reference material value assigned by dPCR, whereas the vertical bars represent the standard deviation based on eight ddPCR replicates

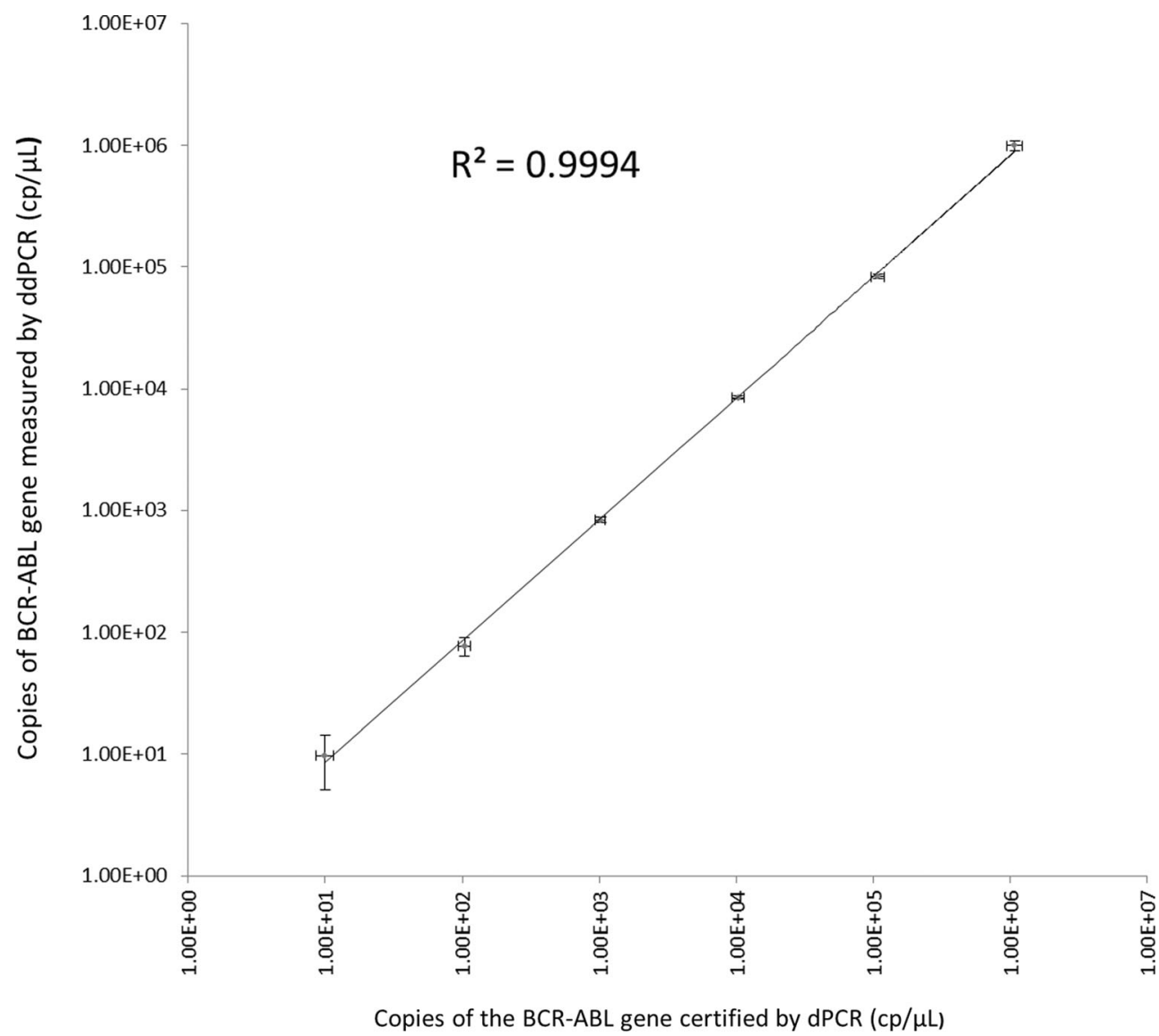

In the 2D image analysis step, two approaches were followed to access the 3D droplet volume (see "Materials and methods" section). The average Feret diameter of the droplets was $3.4 \%$ larger than the diameter calculated using the area equivalent diameter. This observation was explained by noting that the measured Feret diameters are affected by small artificial deviations or tiny protrusions on the droplet perimeter caused by the digital processing of the droplet images. This is the reason why the Feret diameter is consistently larger than the major axis of the best fitting ellipse, and why the area equivalent diameter was chosen to estimate the diameter of the droplets.

The average volume of the droplets generated by the droplet generator in the DG8-186-3008 cartridge (Table 4) was measured as $0.834 \mathrm{~nL}$ which is much smaller than the value of 0.91 nL used in the QuantaSoft software version 1.3.2.0 to calculate the copy number concentration.

Similar experiments have been performed at the NMIA in Sydney reporting volumes of 0.833 and $0.830 \mathrm{~nL}$ using

Table 3 Comparison between the certified copy number concentrations and concentration estimated by ddPCR in the ERM-AD623 series. A droplet volume of $0.91 \mathrm{~nL}$ has been used to calculate the copy number concentration

\begin{tabular}{llllllllll}
\hline ERM-AD623 & $\begin{array}{l}\text { Certified value } \\
(\mathrm{cp} / \mu \mathrm{L})\end{array}$ & $U_{\mathrm{CRM}}$ & $\begin{array}{l}\text { Average measured } \\
\text { values }(\mathrm{cp} / \mu \mathrm{L}), n=8\end{array}$ & stdev & $\begin{array}{l}\Delta_{\mathrm{m}} \\
(\mathrm{cp} / \mu \mathrm{L})\end{array}$ & $\begin{array}{l}u_{\mathrm{m}} \\
(\mathrm{cp} / \mu \mathrm{L})\end{array}$ & $\begin{array}{l}U_{\Delta} \\
(\mathrm{cp} / \mu \mathrm{L})\end{array}$ & $\begin{array}{l}\text { Significant } \\
\text { bias }\end{array}$ & $\begin{array}{l}\% \text { difference } \\
\mathrm{f}\end{array}$ \\
\hline $\mathrm{e}$ & 10 & 1.5 & 9 & 2 & 1 & 1 & 2 & No & 12 \\
$\mathrm{~d}$ & 104 & 10 & 87 & 10 & 17 & 7 & 14 & Yes & 16 \\
$\mathrm{c}$ & 1020 & 90 & 846 & 80 & 174 & 60 & 121 & Yes & 17 \\
$\mathrm{~b}$ & 10,300 & 1000 & 8851 & 669 & 1449 & 602 & 1203 & Yes & 14 \\
$\mathrm{a}$ & 108,000 & 11,000 & 90,951 & 8105 & 17,049 & 6832 & 13,663 & Yes & 16 \\
\hline
\end{tabular}

The calculated bias is significant with a confidence level of $95 \%$

$U_{C R M}$ expanded uncertainty of the certified value; $\Delta_{m}$ absolute difference between mean measured value and certified value; $U_{\Delta}$ combined uncertainty of result and certified value; $u_{m}$ uncertainty of the measurement result, calculated as the standard deviation divided by the square root of the number of replicates 


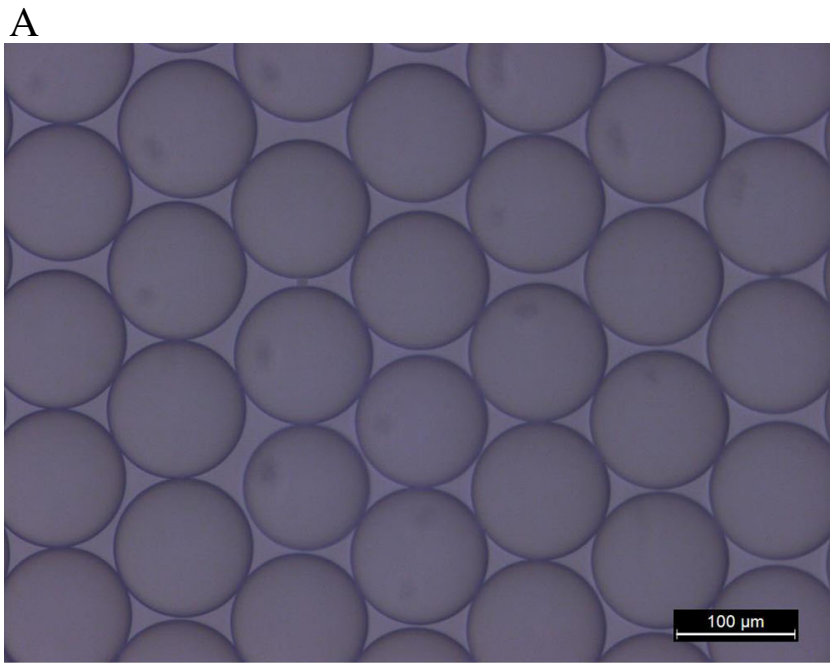

B

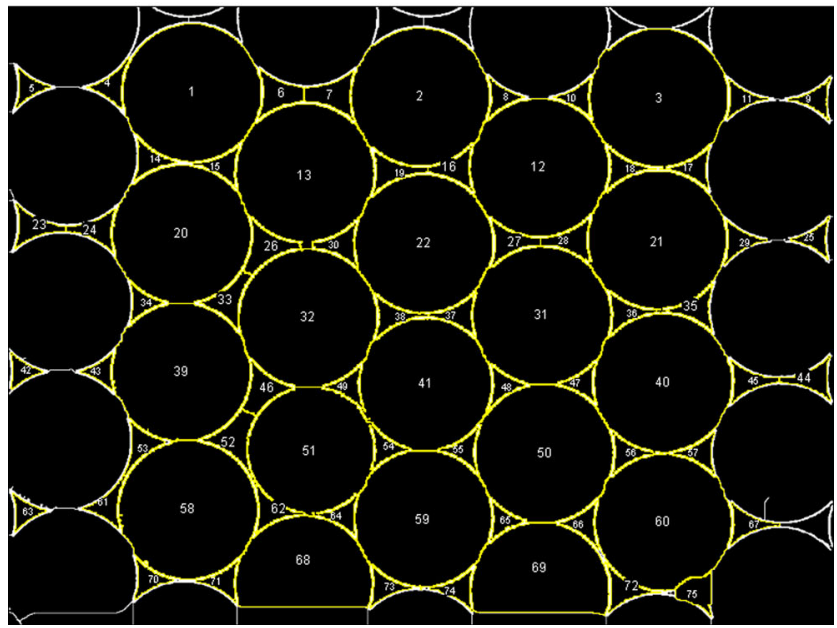

Fig. 3 Typical image taken of a monolayer of droplet generated by the droplet generator and observed under an optical microscope (A) as well as the image treated by the ImageJ software (v1.47q) to distinguish the droplets from each other and to differentiate the in-between droplet areas from the droplet areas $(\mathbf{B})$

ddPCR ${ }^{\mathrm{TM}}$ Supermix for Probes and QX200 ${ }^{\mathrm{TM}} \mathrm{dPCR}^{\mathrm{TM}}$ EvaGreen ${ }^{\circledR}$ Supermix, respectively (Table 5). To assess the uniformity of the droplet volume within a well, 16 sequential $2 \mu \mathrm{L}$ samples were collected from a single well of a DG8 cartridge. The relative expanded uncertainty across the entire

Table 4 Average area equivalent diameters and corresponding volumes of droplets generated by the Bio-Rad Droplet Generator using DG8-1863001 cartridges. The estimated copy number concentrations of ERM$\mathrm{AD} 623 \mathrm{c}$ in the $B C R-A B L$ ddPCR assay were calculated using the
16 sub-samples from a single well was $2.4 \%(k=2)$ (Table 5$)$, indicating little variability and good within-well precision. The similarity in values reported from two different laboratories demonstrates good agreement and reproducibility between laboratories applying the same methodology, but using different lots of cartridges and different master mixes.

The relative expanded uncertainty associated to the average droplet volume estimated by IRMM and NMIA for the ddPCR $^{\text {TM }}$ Supermix for Probes was 3.6 \% $(k=2)$ and $4.2 \%$ $(k=2)$, respectively, indicating an appreciable small variation in the volume of the droplets. For the QX200 ${ }^{\mathrm{TM}} \mathrm{ddPCR}^{\mathrm{TM}}$ EvaGreen ${ }^{\circledR}$ Supermix, a very similar relative expanded uncertainty associated to the average droplet volume [3.3\% $(k=2)]$ was estimated indicating also only a small variation of the volume of the droplets with those two different master mixes. On some images, noticeable smaller droplets were observed (results not shown), but according to the manufacturer, those very small droplets are eliminated by the reader and are not used by the software as countable droplets.

\section{Discussion}

The droplet volume measured using the DG8 cartridge is slightly smaller than the volume generated using the betaversion of the droplet generator [29] demonstrating the importance of re-measuring the droplet volume when new generators or cartridges are released. Equation (1) illustrates that the copy number concentration is inversely related to the average droplet volume $\left(V_{\mathrm{d}}\right)$. Hence, if the number of positive droplets $(H)$ and total number of droplets $(C)$ are constant, the use of a smaller droplet volume $\left(V_{\mathrm{d}}\right)$ in the formula will increase the estimated copy number concentration. This is illustrated in Fig. 4, showing how the estimated copy number concentration per microliter of the plasmid in ERM-AD623c varies depending on the droplet volume $\left(V_{\mathrm{d}}\right)$ used in the equation.

The copy number concentration measured by ddPCR for the ERM-AD623c was $9454 \mathrm{cp} / \mu \mathrm{L}$ with an expanded uncertainty of $200 \mathrm{cp} / \mu \mathrm{L}$ taking into consideration the measured droplet volume of $0.834 \mathrm{~nL}$ in our experimental setup

corresponding droplet volume that was estimated each day, together with its standard deviation. The uncertainty on the average copy number concentration is an expanded uncertainty with a coverage factor $k$ of 2

\begin{tabular}{llll}
\hline & Average area equivalent diameter $(\mu \mathrm{m})$ & Droplet volume $(\mathrm{nL})$ & Average copy number concentration $(\mathrm{cp} / \mu \mathrm{L})$ \\
\hline Day 1 & $117.02 \pm 1.37(n=588)$ & $0.839 \pm 0.03(n=588)$ & $9310 \pm 196(n=8)$ \\
Day 2 & $116.59 \pm 2.21(n=605)$ & $0.830 \pm 0.04(n=605)$ & $9586 \pm 224(n=8)$ \\
Day 3 & $116.60 \pm 3.18(n=601)$ & $0.830 \pm 0.06(n=601)$ & $9465 \pm 352(n=8)$ \\
Average & & 0.834 & $9454 \pm 200(k=2)$ \\
\hline
\end{tabular}


Table 5 Average volume of droplets generated by the Bio-Rad Droplet Generator using either Supermix for Probes or EvaGreen Supermix. The uncertainty on the average copy number concentration is an expanded uncertainty with a coverage factor $k$ of 2

\begin{tabular}{llll}
\hline Cartridge & Mastermix & $\begin{array}{l}\text { Droplet volume } \\
(\mathrm{nL})\end{array}$ & $\begin{array}{l}\text { Total number of } \\
\text { droplets }\end{array}$ \\
\hline DG8 (production year 2012) & & $0.833 \pm 0.035$ & 622 \\
$\begin{array}{l}\text { DG8 (lot nos. C000021590, } \\
\quad \text { C00024241 and C000031616) }\end{array}$ & ddPCR $^{\mathrm{T}}$ Supermix for Probes & $0.830 \pm 0.027$ & 805 \\
DG8 (production year 2013) & QX200 & \\
\hline
\end{tabular}

${ }^{a}$ Droplets were analysed from three wells from each of five DG8 cartridges (15 wells in total)

${ }^{\mathrm{b}}$ Sixteen $2 \mu \mathrm{L}$ samples were collected sequentially from droplets generated from a single well of a DG8 cartridge and the average droplet volume measured

(Table 4). The uncertainty associated to the average copy number concentration was determined taking into account the variance of the repeatability estimated by the residual of the ANOVA and the variance of the between-day repeatability or the intermediate precision. The certified concentration of $10,300 \mathrm{cp} / \mu \mathrm{L}$ remains slightly higher than the value measured by ddPCR. However, the remaining bias of $8 \%$ is statistically no longer significant as it is smaller than the combined uncertainty of the certified value and the measured value.

The bias observed in the first set of experiments using the manufacture-specified droplet volume of $0.91 \mathrm{~nL}$ (Fig. 4(a)) disappears when taking into account the real droplet volume as determined in this study (Fig. 4(c)). Indeed, the measured copy number concentration taking into account the real droplet volume increased by $8 \%$ bringing it closer to the certified values assigned by dPCR. When applying the measured volume of $0.834 \mathrm{~nL}$ to recalculate the copy number concentrations reported in Table 3 , the bias observed for the values on the ERM-AD623 series is not statistically significant

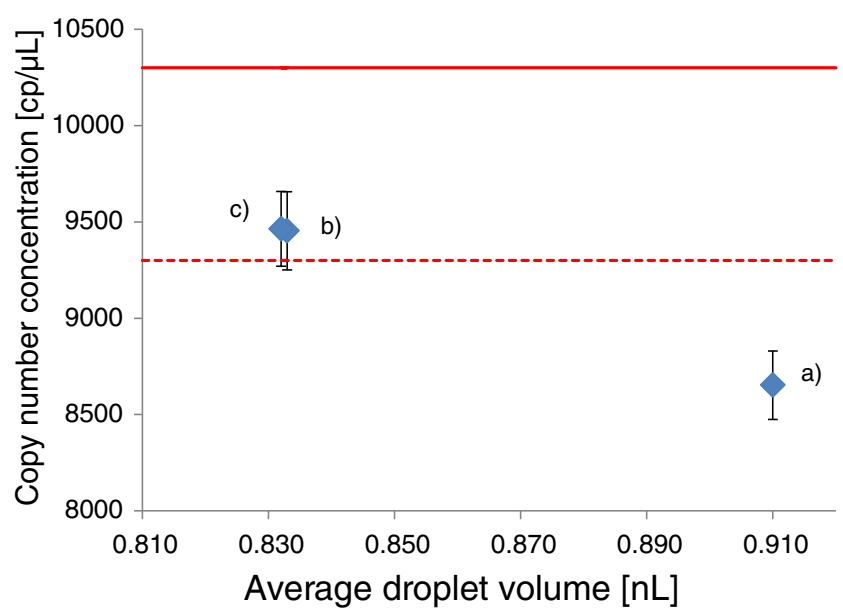

Fig. 4 Influence of the value used for average droplet volume in Eq. (1) on the copy number concentration (displayed with their standard deviation) determined by ddPCR. (a) With using a volume of $0.91 \mathrm{~nL}$ used in the QuantaSoft version 1.3.2.0, (b) with using an average volume of $0.834 \mathrm{~nL}$ measured in this study and (c) with using an average volume of $0.833 \mathrm{~nL}$ measured by NMIA. The continuous line represents the certified value assigned by dPCR and the dotted line displays the $95 \%$ lower confidence interval anymore. The copy number concentrations determined by ddPCR remain nevertheless slightly lower (between 4 and $9 \%$ ) than those reported by dPCR. This could be attributed to a variability in the partition volume in the Fluidigm 12.765 digital array, since the relative standard uncertainty of the average partition volume for the 12.765 digital array has previously been estimated at $5 \%$ [27]. More extensive studies to verify the accuracy of the partition volume in $\mathrm{APCR}$ would be required to exclude this possibility.

The finding of two independent laboratories concerning the droplet volume generated by the DG8-186-3008 has been
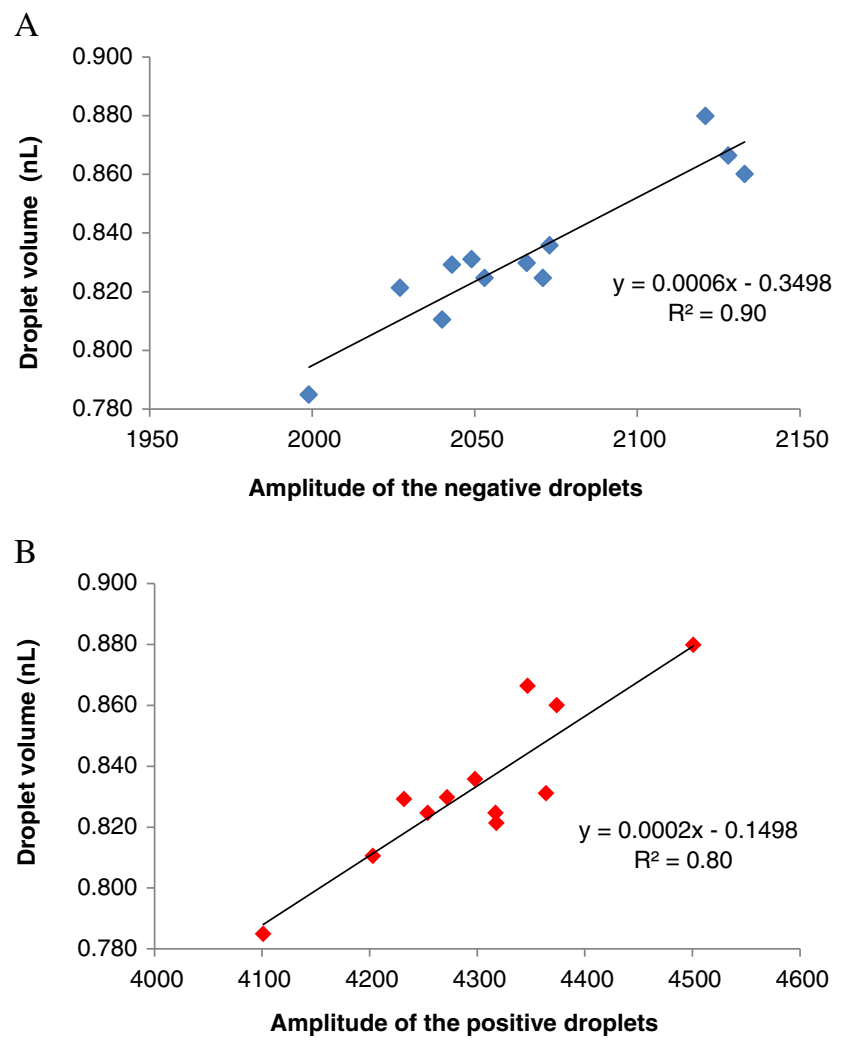

Fig. 5 Relationship between the average amplitude of the fluorescence in negative (A) and positive (B) droplets in dependence on the droplet size. Each diamond represents the average droplet volume and the average fluorescence amplitude of droplets generated from a single well in a droplet generator cartridge 
communicated to the instrument producer Bio-Rad, who has since launched a newer version of the QuantaSoft software (version 1.6.6) using an average droplet volume of $0.85 \mathrm{~nL}$. This newer software version coincides with the placing on the market of a newer droplet generator cartridge (DG8-1864008) that is supposed to replace the earlier cartridges [BioRad, personal communication]. The value of $0.85 \mathrm{~nL}$ used in the software version 1.6.6 is not accompanied with an uncertainty but is lowered enough to avoid the significant bias observed with the earlier software version. No further details on the method used to determine this volume could be obtained from Bio-Rad.

The variability in the volume of droplets generated from different cartridge wells and across different channels correlated with changes in the average fluorescence amplitude reported by the QuantaSoft software (Fig. 5A, B). A slightly stronger fluorescence signal for both positive and negative droplets was observed in larger droplets. This is not unexpected since larger droplets will contain more fluorescence molecules than smaller droplets. It means that a small variation of the fluorescence amplitude among replicates of the same assay (around $10 \%$ in this study) is an indication that droplets with different average volumes were produced in each channel of the droplet generator. In an optimised assay, it is theoretically possible to recalculate the copy number concentration with a correction factor that takes into account the variability of the droplet volume among replicates. This feature may be exploited in the future to improve the precision of ddPCR measurements.

\section{Conclusion}

In this study, a reference material certified for the copy number concentration of a plasmid solution over a concentration range from 1 million to $10 \mathrm{cp} / \mu \mathrm{L}$ was used to compare the certified values determined by dPCR with results from another digital PCR method based on droplet emulsion. Copy number concentrations measured by those two digital PCR methods were in good agreement, if the real average volume of the droplets was used to recalculate the assay volume. The volume of $0.91 \mathrm{~nL}$ used in the QuantaSoft software version 1.3.2.0 was found to be inaccurate and was the source of the bias observed between the results using the two digital PCR platforms. This study illustrates on the benefit of using a certified reference material to validate methods and procedures involving digital PCR and the risk of relying on data generated by a software which makes assumptions on the value of one or more experimental variables.

For many clinical applications, where copy numbers are expressed as ratios, the uncertainty on the volume - provided the volume stays constant - is not so critical. However, when digital PCR is used to assign 'absolute' copy number concentrations, the volume of the assay needs ideally to be confirmed. The droplet volume of $0.85 \mathrm{~nL}$ used in the latest software version from Bio-Rad is a value that is very close to the average droplet volume measured in this paper. Assuming that other parameters that affect the number of positive droplets, the total number of droplets and the dilution process are under control, an 'absolute' quantification of copy number using that pre-set volume can be reliable. However, digital PCR-based enumeration techniques to quantify bacteria, viruses or whole cells that are encapsulated in the droplets from which the volume is unknown or fluctuating with the applied experimental conditions will require the real volume of the droplets to be taken into consideration $[33,34]$.

Open Access This article is distributed under the terms of the Creative Commons Attribution License which permits any use, distribution, and reproduction in any medium, provided the original author(s) and the source are credited.

\section{References}

1. Raso A, Biassoni R (2014) Twenty years of qPCR: a mature technology? Methods Mol Biol 1160:1-3

2. VanGuilder HD, Vrana KE, Freeman WM (2008) Twenty-five years of quantitative PCR for gene expression analysis. Biotechniques 44: 619-626

3. Klatte M, Bauer P (2009) Accurate real-time reverse transcription quantitative PCR. Methods Mol Biol 479:61-77

4. Lee SB, McCord B, Buel E (2014) Advances in forensic DNA quantification: a review. Electrophoresis. doi:10.1002/elps.201400187

5. Horsman KM, Bienvenue JM, Blasier KR, Landers JP (2007) Forensic DNA analysis on microfluidic devices: a review. J Forensic Sci 52:784-799

6. Hall Sedlak R, Jerome KR (2014) The potential advantages of digital PCR for clinical virology diagnostics. Expert Rev Mol Diagn 14: 501-507

7. Sedlak RH, Jerome KR (2013) Viral diagnostics in the era of digital polymerase chain reaction. Diagn Microbiol Infect Dis 75(1):1-4

8. Kumar A, Rahal A, Chakraborty S, Verma AK, Dhama K (2014) Mycoplasma agalactiae, an etiological agent of contagious agalactia in small ruminants: a review. Vet Med Int. doi:10.1155/2014/286752

9. Beer (1852) Bestimmung der Absorption des rothen Lichts in farbigen Flüssigkeiten. Ann Phys Chem 86:78-88

10. Singer VL, Jones LJ, Yue ST, Haugland RP (1997) Characterization of PicoGreen reagent and development of a fluorescence-based solution assay for double-stranded DNA quantitation. Anal Biochem 249: 228-238

11. Bowers RM, Dhar AK (2011) Effect of template on generating a standard curve for absolute quantification of an RNA virus by realtime reverse transcriptase-polymerase chain reaction. Mol Cell Probes 25:60-64

12. Chen J, Kadlubar FF, Chen JZ (2007) DNA supercoiling suppresses real-time PCR: a new approach to the quantification of mitochondrial DNA damage and repair. Nucleic Acids Res 35:1377-1388

13. Hou Y, Zhang H, Miranda L, Lin S (2010) Serious overestimation in quantitative PCR by circular (supercoiled) plasmid standard: microalgal pcna as the model gene. PLoS One 5:e9545

14. Wu YL, Savelli SL, Yang Y, Zhou B, Rovin BH, Birmingham DJ, Nagaraja HN, Hebert LA, Yu CY (2007) Sensitive and specific real- 
time polymerase chain reaction assays to accurately determine copy number variations (CNVs) of human complement C4A, C4B, C4long, C4-short, and RCCX modules: elucidation of $\mathrm{C} 4 \mathrm{CNVs}$ in 50 consanguineous subjects with defined HLA genotypes. J Immunol 179:3012-3025

15. Øvstebø R, Haug KB, Lande K, Kierulf P (2003) PCR-based calibration curves for studies of quantitative gene expression in human monocytes: development and evaluation. Clin Chem 49:425-432

16. Toyota A, Akiyama H, Sugimura M, Watanabe T, Sakata K, Shiramasa Y, Kitta K, Hino A, Esaka M, Maitani T (2006) Rapid quantification methods for genetically modified maize contents using genomic DNAs pretreated by sonication and restriction endonuclease digestion for a capillary-type real-time PCR system with a plasmid reference standard. Biosci Biotechnol Biochem 70:2965-2973

17. Yukl SA, Kaiser P, Kim P, Li P, Wong JK (2014) Advantages of using the QIAshredder instead of restriction digestion to prepare DNA for droplet digital PCR. Biotechniques 56:194-196

18. Sivaganesan M, Seifring S, Varma M, Haugland RA, Shanks OC (2008) A Bayesian method for calculating real-time quantitative PCR calibration curves using absolute plasmid DNA standards. BMC Bioinforma 9:120

19. Burns M, Corbisier P, Wiseman G, Valdivia H, McDonald P, Bowler P, Ohara K, Schimmel H, Charels D, Damant A, Harris N (2006) Comparison of plasmid and genomic DNA calibrants for the quantification of genetically modified ingredients. Eur Food Res Technol 224:249-258

20. Caprioara-Buda M, Meyer W, Jeynov B, Corbisier P, Trapmann S, Emons H (2012) Evaluation of plasmid and genomic DNA calibrants used for the quantification of genetically modified organisms. Anal Bioanal Chem 404:29-42

21. Corbisier P, Vincent S, Schimmel H, Kortekaas AM, Trapmann S, Burns M, Bushell C, Akgoz M, Akyürek S, Dong L, Fu B, Zhang L, Wang J, Pérez Urquiza M, Bautista JL, Garibay A, Fuller B, Baoutina A, Partis L, Emslie K, Holden M, Chum WY, Kim HH, Phunbua N, Milavec M, Zel J, Vonsky M, Konopelko LA, Lau TLT, Yang B, Hui MHK, Yu ACH, Viroonudomphol D, Prawettongsopon C, Wiangnon K, Takabatake R, Kitta K, Kawaharasaki M, Parkes H (2012) CCQM-K86/P113.1: relative quantification of genomic DNA fragments extracted from a biological tissue. Metrologica 1A (Technical Supplement) vol 49

22. Vogelstein B, Kinzler KW (1999) Digital PCR. Proc Natl Acad Sci U S A 96:9236-9241

23. Haynes RJ, Kline MC, Toman B, Scott C, Wallace P, Butler JM, Holden MJ (2013) Standard reference material 2366 for measurement of human cytomegalovirus DNA. J Mol Diagn 15:177-185

24. Deprez L, Mazoua S, Corbisier P, Trapmann S, Schimmel H, White H, Emons H (2012) The certification of the copy number concentration of solutions of plasmid DNA containing a BCR-ABL b3a2 transcript fragment. Certified reference material: ERM-AD623a, ERM-AD623b, ERM-AD623c, ERM-AD623d, ERM-AD623e,
ERM-AD623f. Luxembourg: Publications Office of the European Union, 2012; Report number EUR 25248; ISBN 978-92-79-23343-2

25. Sanders R, Mason DJ, Foy CA, Huggett JF (2013) Evaluation of digital PCR for absolute RNA quantification. PLoS One 8:e75296

26. Dong L, Meng Y, Wang J, Liu Y (2014) Evaluation of droplet digital PCR for characterizing plasmid reference material used for quantifying ammonia oxidizers and denitrifiers. Anal Bioanal Chem 406: $1701-1712$

27. Bhat S, Herrmann J, Armishaw P, Corbisier P, Emslie KR (2009) Single molecule detection in nanofluidic digital array enables accurate measurement of DNA copy number. Anal Bioanal Chem 394: $457-467$

28. Huggett JF, Foy CA, Benes V, Emslie K, Garson JA, Haynes R, Hellemans J, Kubista M, Mueller RD, Nolan T, Pfaffl MW, Shipley GL, Vandesompele J, Wittwer CT, Bustin SA (2013) Guidelines for minimum information for publication of quantitative digital PCR experiments. Clin Chem 59:892-902

29. Pinheiro LB, Coleman VA, Hindson CM, Herrmann J, Hindson BJ, Bhat S, Emslie KR (2012) Evaluation of a droplet digital polymerase chain reaction format for DNA copy number quantification. Anal Chem 84:1003-1011

30. White H, Deprez L, Corbisier P, Hall V, Lin F, Mazoua S, Trapmann S, Aggerholm A, Andrikovics H, Akiki S, Barbany G, Boeckx N, Bench A, Catherwood M, Cayuela JM, Chudleigh S, Clench T, Colomer D, Daraio F, Dulucq S, Farrugia J, Fletcher L, Foroni L, Ganderton R, Gerrard G, Gineikienė E, Hayette S, El Housni H, Izzo B, Jansson M, Johnels P, Jurcek T, Kairisto V, Kizilors A, Kim DW, Lange T, Lion T, Polakova KM, Martinelli G, McCarron S, Merle PA, Milner B, Mitterbauer-Hohendanner G, Nagar M, Nickless G, Nomdedéu J, Nymoen DA, Leibundgut EO, Ozbek U, Pajič T, Pfeifer H, Preudhomme C, Raudsepp K, Romeo G, Sacha T, Talmaci R, Touloumenidou T, Van der Velden VH, Waits P, Wang L, Wilkinson E, Wilson G, Wren D, Zadro R, Ziermann J, Zoi K, Müller MC, Hochhaus A, Schimmel H, Cross NC, Emons H (2014) A certified plasmid reference material for the standardisation of BCRABL1 mRNA quantification by real-time quantitative PCR. Leukemia. doi:10.1038/leu.2014.217

31. ISO 13322-1 (2004) Particle size analysis - image analysis methods - part 1: static image analysis methods

32. Linsinger T (2010) ERM Application note 1: comparison of a measurement result with the certified value https://ec.europa.eu/jrc/sites/ default/files/erm application note 1 en.pdf. Accessed 11 Nov 2014

33. Zeng Y, Novak R, Shuga J, Smith MT, Mathies RA (2010) Highperformance single cell genetic analysis using microfluidic emulsion generator arrays. Anal Chem 82:3183-3190

34. Dreo T, Pirc M, Ramšak Ž, Pavšič J, Milavec M, Zel J, Gruden K (2014) Optimising droplet digital PCR analysis approaches for detection and quantification of bacteria: a case study of fire blight and potato brown rot. Anal Bioanal Chem 406:6513-6528 